Learning objectives

This pictorial review aims to:

- Describe the use of cross sectional imaging in the evaluation of acute, chronic and autoimmune pancreatitis and their common imaging findings.

- Imaging review of most frequently encountered complications of pancreatitis.

Intrerstitial Oedematous Pancreatitis
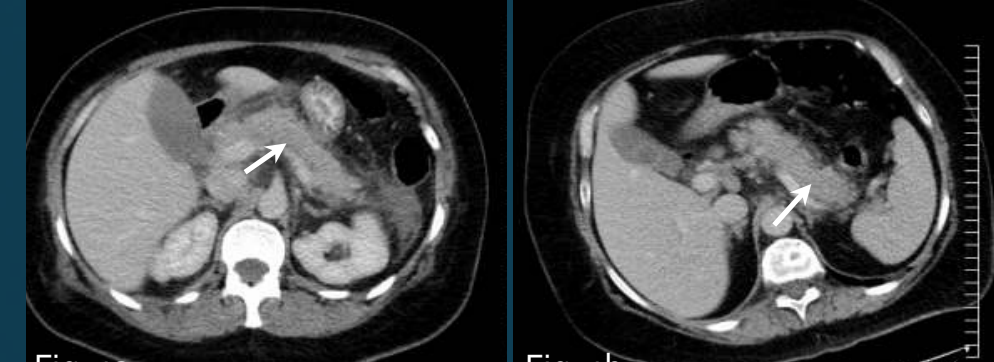

Acute Peripancreatic Fluid Collection

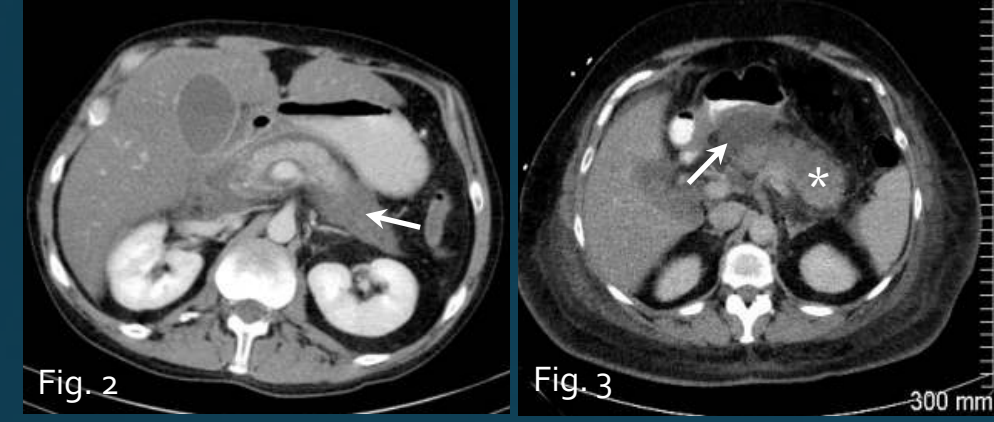

Pancreatic Pseudocyst

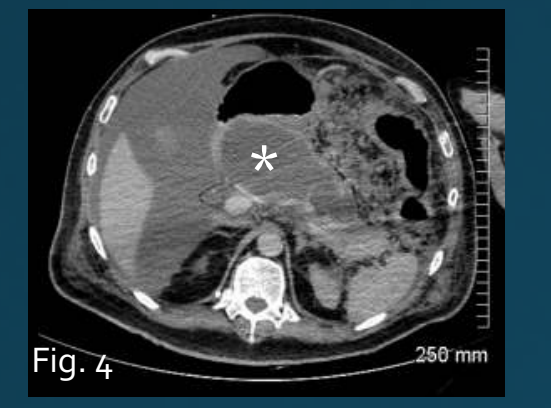

Acute Necrosis

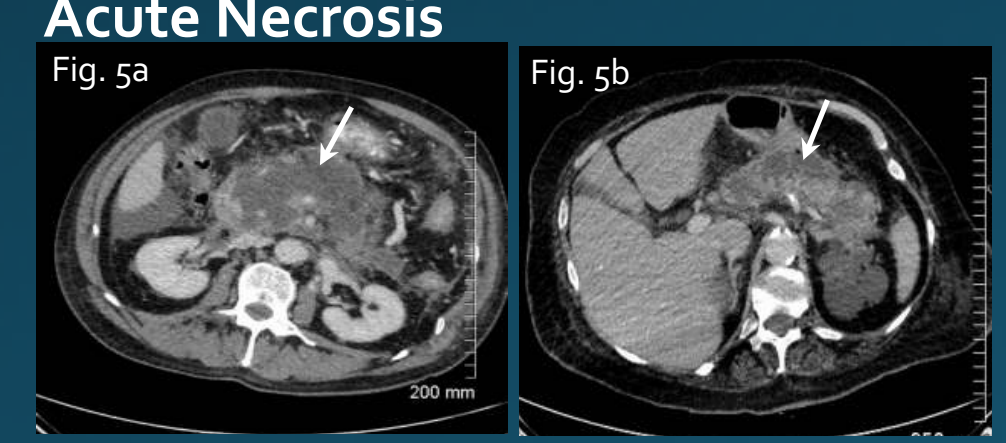

Walled-Off Necrosis (WON)

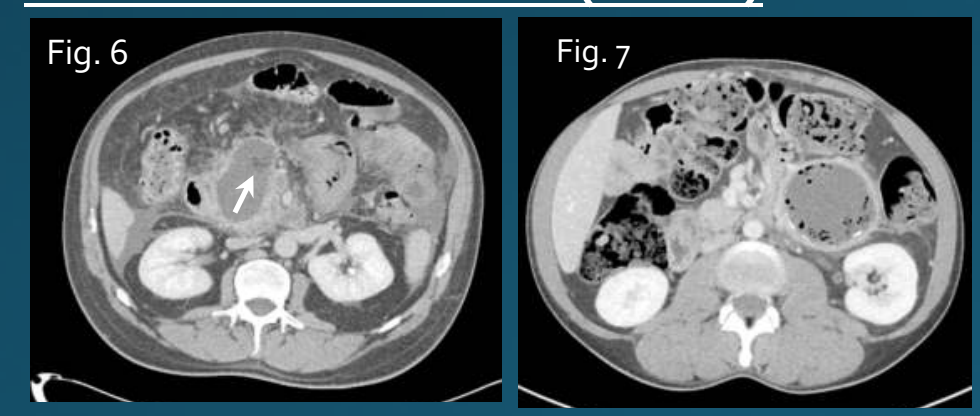

Complications of Acute Pancreatitis

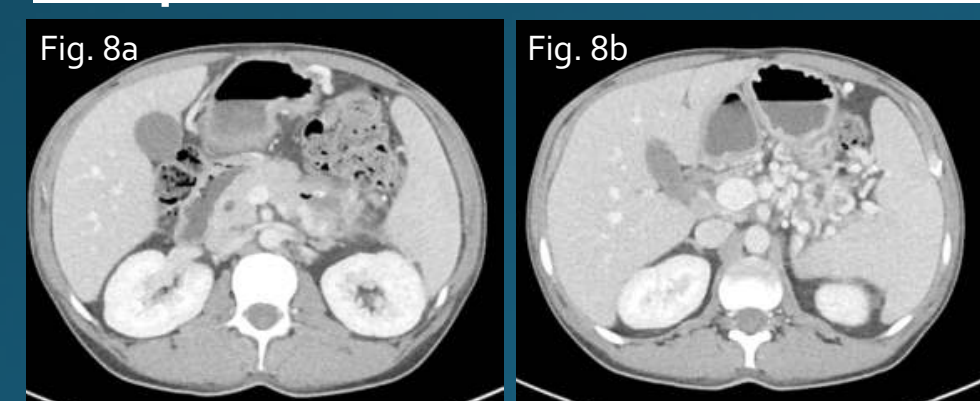

Acute Pancreatitis - Revised Atlanta Classification 2012

Interstitial Oedematous Pancreatitis (90\%)

Necrotizing Pancreatitis (5-10\%)

- Acute peripancreatic fluid collection (APFC) ( $<4$ weeks)

- Pancreatic pseudocyst (> 4 weeks)

- Acute necrotic collection (ANC) $(<4$ weeks)

- Walled - off necrosis (WON) (> 4 weeks)

\section{Chronic Pancreatitis}

Types of Chronic Pancreatitis

- Chronic calcifying pancreatitis

- Chronic obstructive pancreatitis

- Autoimmune pancreatitis

- Groove pancreatitis

Figure 1. Diffuse pancreatic enlargement and oedema (arrowed) with peripancreatic fat stranding. Diffuse enhancement of the pancreatic parenchyma with no evidence of focal necrosis.

Figure 2. Homogeneous fluid attenuation collection at the left anterior pararenal space. Normal enhancement of the pancreatic parenchyma with no focal necrosis.

Figure 3. Postcontrast hypoenhancement of the pancreatic body in keeping with early pancreatic necrosis (asterisk). III defined fluid collection separate from the pancreatic neck (arrow) consistent with peripancreatic fluid collection.

Figure 4. Axial CT 6 weeks following onset of acute pancreatitis shows a large homogeneous encapsulated fluid collection (asterisk) in the lesser sac with enhancing walls, consistent with a pseudocyst.

Figure 5. Axial postcontrast CT obtained 2 weeks post onset of symptoms demonstrates diffuse parenchymal hypoenhancement with surrounding inflammatory change, in keeping with acute necrosis.

Figure 6. Axial portal venous phase $\mathrm{CT}$ abdomen shows a peripherally enhancing lesion, of fluid attenuation arising from the pancreatic neck. Intraluminal fat debris (arrowed) is in keeping with walled-off necrosis in comparison with a pseudocyst (no debris).

Figure 7. WON with intraluminal gas and debris

Figure 8. Splenic vein obstruction (8a) with resulting well formed splenic collateral circulation (8b)
Chronic Calcifying Pancreatitis

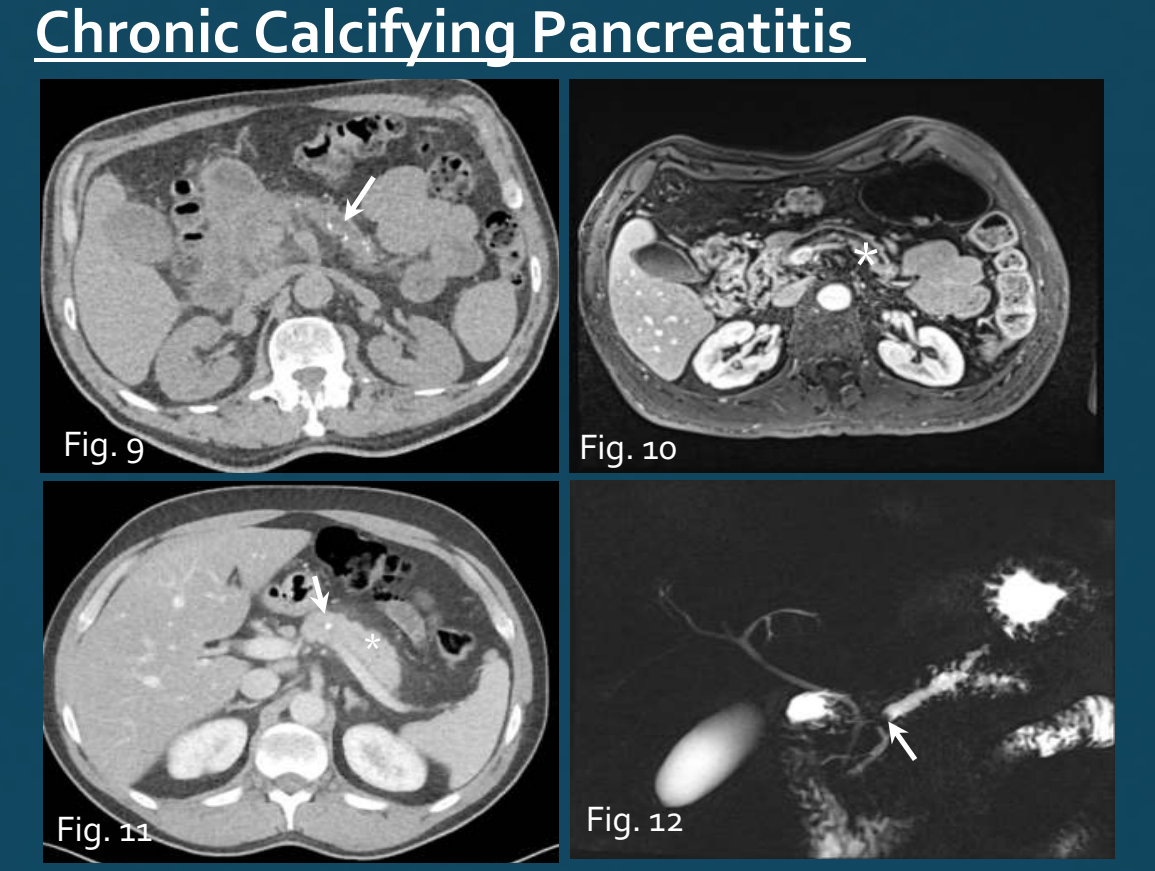

Chronic Obstructive Pancreatitis

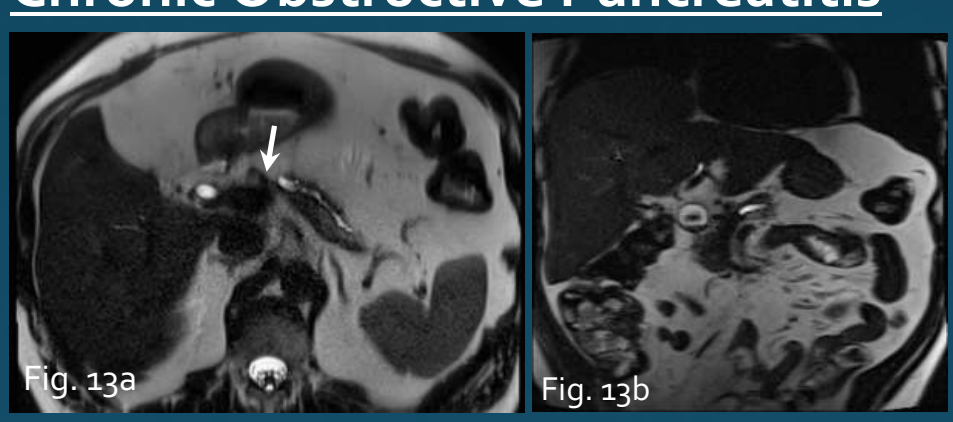

Acute Autoimmune Pancreatitis

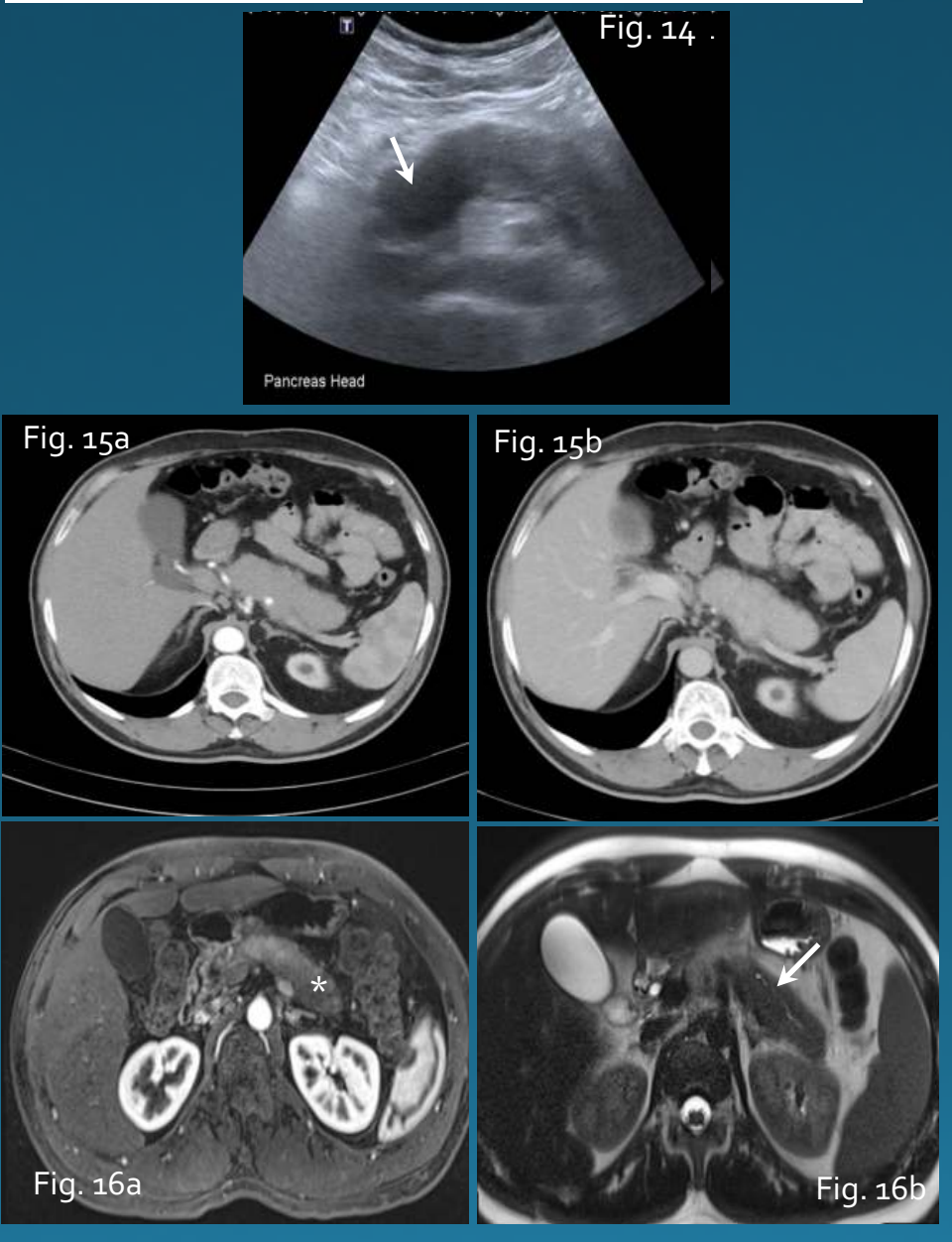

Autoimmune Pancreatitis (AIP) Imaging Features

- Delayed enhancement on portal venous phase on CT abdomen

- Tapered pancreatic duct occlusion/ stenosis vs abrupt occlusion in adenocarcinoma

- Diffuse hypertrophy of the entire gland

Unlikely inflammatory change or adenopathy

Figure 9. Portal venous phase $\mathrm{CT}$ abdomen shows pancreatic tail atrophy, multiple intraparenchymal calcifications and a dilated pancreatic duct (arrowed)

Figure 10. T1 post contrast MR Imaging shows an atrophic pancreatic tail with a dilated pancreatic duct (asterisk) in keeping with chronic pancreatitis.

Figure 11. Portal venous phase $C T$ demonstrates atrophic pancreatic parenchyma with intraductal calcifications (arrowed) and resultant pancreatic duct dilatation (asterisk).

Figure 12. Coronal MIP MRCP images show a markedly dilated pancreatic duct with cystic dilatation of the side branches secondary to a filling defect within the distal portion (arrowed), in keeping with an intraductal calculus and resultant obstruction.

Figure 13. Pancreatic atrophy with pancreatic neck fibrosis (arrowed) as sequela of acute necrosis as visualised on axial (a) and coronal (b) T2 HASTE slices. Resultant pancreatic duct obstruction with irregularities of the main pancreatic duct contour.

Figure 14. Diffusely enlarged pancreatic parenchyma with a hypoechoic sonographic appearance, characteristic for autoimmune pancreatitis

Figure 15. CT arterial and portal venous phase contrast enhanced pancreatic imaging shows a diffusely enlarged, mass like, pancreatic tail with loss of folia definition and a halo of soft tissue attenuation (thought to represent inflammatory lymphocytic cell infiltration).

Figure 16. Early venous phase contrast enhanced $\mathrm{T}_{1}$ Fat Sat MR (a) shows delayed enhancement within the affected pancreatic tail (asterisk) when compared to the normal pancreatic neck. Hypointense pancreas on T2 HASTE MR (b). Pancreatic duct irregularity secondary to parenchymal mass effect (arrowed) demonstrated on the T2 HASTE MR axial image. Note no pancreatic duct dilatation proximal to the stricture. 